\title{
Genetic diversity of Plasmodium vivax and Plasmodium falciparum lactate dehydrogenases in Myanmar isolates
}

Jinyoung Lee ${ }^{1}$, Tae Im Kim 2,6, Hương Giang Lê2,3, Won Gi Yoo ${ }^{4}$, Jung-Mi Kang ${ }^{2,3}$, Seong-Kyu Ahn', Moe Kyaw Myint ${ }^{5}$, Khin Lin ${ }^{5}$, Tong-Soo Kim ${ }^{1 *}$ and Byoung-Kuk Na $2,3^{*}$

\begin{abstract}
Background: Plasmodium lactate dehydrogenase (pLDH) is a major target in diagnosing the erythrocytic stage of malaria parasites because it is highly expressed during blood-stage parasites and is distinguished from human LDH. Rapid diagnostic tests (RDTs) for malaria use pLDH as a target antigen; however, genetic variations in pLDH within the natural population threaten the efficacy of pLDH-based RDTs.

Methods: Genetic polymorphisms of Plasmodium vivax LDH (PvLDH) and Plasmodium falciparum LDH (PfLDH) in Myanmar isolates were analysed by nucleotide sequencing analysis. Genetic polymorphisms and the natural selection of PVLDH and PfLDH were analysed using DNASTAR, MEGA6, and DnaSP ver. 5.10.00 programs. The genetic diversity and natural selection of global PVLDH and PfLDH were also analysed. The haplotype network of global PVLDH and PfLDH was constructed using NETWORK ver. 5.0.0.3. Three-dimensional structures of PvLDH and PfLDH were built with YASARA Structure ver. 18.4.24 and the impact of mutations on structural change and stability was evaluated with SDM ver. 2, CUPSAT and MAESTROweb.

Results: Forty-nine PVLDH and 52 PfLDH sequences were obtained from Myanmar P. vivax and P. falciparum isolates. Non-synonymous nucleotide substitutions resulting in amino acid changes were identified in both Myanmar PVLDH and PfLDH. Amino acid changes were also identified in the global PvLDH and PfLDH populations, but they did not produce structural alterations in either protein. Low genetic diversity was observed in global PVLDH and PfLDH, which may be maintained by a strong purifying selection.

Conclusion: This study extends knowledge for genetic diversity and natural selection of global PVLDH and PfLDH. Although amino acid changes were observed in global PVLDH and PfLDH, they did not alter the conformational structures of the proteins. These suggest that PVLDH and PfLDH are genetically well-conserved in global populations, which indicates that they are suitable antigens for diagnostic purpose and attractive targets for drug development.
\end{abstract}

Keywords: Plasmodium vivax, Plasmodium falciparum, Lactate dehydrogenase, Myanmar, Genetic diversity

*Correspondence: tongsookim@inha.ac.kr; bkna@gnu.ac.kr ${ }^{1}$ Department of Tropical Medicine and Inha Research Institute for Medical Science, Inha University School of Medicine, Incheon, Republic of Korea

${ }^{2}$ Department of Parasitology and Tropical Medicine, and Institute of Health Sciences, Gyeongsang National University College of Medicine, Jinju 52727, Republic of Korea

Full list of author information is available at the end of the article

\section{Background}

Malaria is an acute febrile infectious disease caused by Plasmodium parasites and is widely endemic in tropical and subtropical areas. Although the global incidence and mortality of malaria have decreased in recent years, malaria is still a major public health concern with an estimated 219 million cases causing 435,000 deaths in 2017 [1]. Despite advances in malaria

c) The Author(s) 2020. This article is licensed under a Creative Commons Attribution 4.0 International License, which permits use, sharing, adaptation, distribution and reproduction in any medium or format, as long as you give appropriate credit to the original author(s) and the source, provide a link to the Creative Commons licence, and indicate if changes were made. The images or other third party material in this article are included in the article's Creative Commons licence, unless indicated otherwise in a credit line to the material. If material is not included in the article's Creative Commons licence and your intended use is not permitted by statutory regulation or exceeds the permitted use, you will need to obtain permission directly from the copyright holder. To view a copy of this licence, visit http://creativeco mmons.org/licenses/by/4.0/. The Creative Commons Public Domain Dedication waiver (http://creativecommons.org/publicdomain/ zero/1.0/) applies to the data made available in this article, unless otherwise stated in a credit line to the data. 
diagnostic methods, microscopic examination of blood smear still remains the gold standard for malaria diagnosis [2]. This method can identify the Plasmodium species and quantify the parasitaemia level at a low cost [1]. However, it is laborious, time-consuming, and requires well-trained and highly qualified technicians. Also, misdiagnoses and incorrect species identification can occur in cases of low parasitaemia, leading to incorrect treatment [3]. To overcome these disadvantages, several alternative methods for malaria diagnosis have been developed.

Rapid diagnostic tests (RDTs) that detect plasmodial antigens circulating in the blood during the infection are the most powerful alternatives to microscopy, especially in conditions where microscopic examination is not feasible [4-6]. RDTs have been used as reliable, massive screening tools in malaria-endemic areas. However, several important issues including diagnostic accuracy, high cost, and reliability in unfavorable field conditions plague RDTs. Several plasmodial antigens including histidine-rich protein 2 (pHRP-2), plasmodial aldolase, and plasmodial lactate dehydrogenase (pLDH) have been used in RDTs. Among them, pHRP-2, especially P. falciparum HRP-2 (PfHRP-2), has been used in numerous RDTs because combination (or combo) RDTs can target antigens for P. falciparum (PfHRP-2) and non-falciparum (or panspecific; pLDH), simultaneously. However, concerns for HRP-2-based RDTs, especially for P. falciparum, have increased in recent years due to false-negative results caused by the deletion of the target gene in malaria parasites [7-12]. Therefore, many manufactures are developing RDTs that can replace PfHRP-2based RDTs.

pLDH is an enzyme essential to a Plasmodium parasite's survival by mediating parasite's anaerobic glycolysis, and it is expressed at high levels during the blood stage $[13,14]$. Moreover, the level of $\mathrm{pLDH}$ in the blood is directly correlated to the parasitaemia level. Due to these advantages, pLDH has been widely examined as a promising diagnostic antigen and has been adopted in many RDTs for malaria. However, few studies have examined pLDH genetic polymorphisms in Plasmodium isolates and the potential influence pLDH genetic variations have on the diagnostic performance of pLDH-based RDTs [15-17]. In this study, the genetic diversity of $P$. falciparum LDH (PfLDH) and $P$. vivax LDH (PvLDH) in Myanmar parasite isolates was investigated. Moreover, the genetic diversity of global PfLDH and PvLDH populations was also analysed to better understand the genetic structures of the two genes in global $P$. falciparum and $P$. vivax populations.

\section{Methods}

\section{Blood samples and ethics}

The blood samples used in this study were obtained from $P$. vivax and $P$. falciparum infected patients with symptomatic uncomplicated cases who resided in towns and villages located in Naung Cho and Pyin Oo Lwin townships in Upper Myanmar in 2015. Malaria infection was confirmed by Giemsa-stained thick and thin blood smear examinations. Prior to treatment, $2 \mathrm{ml}$ of venous blood was collected from each confirmed $P$. falciparum and $P$. vivax infected patients and placed into ethylenediaminetetraacetic acid (EDTA) tubes for further molecular analysis. All $P$. falciparum and $P$. vivax positive samples were further confirmed with polymerase chain reaction (PCR) targeting the $18 \mathrm{~S}$ ribosomal RNA (rRNA) gene $[18,19]$. The use of blood samples in this study was approved by the Ministry of Health, Myanmar (97/Ethics 2015) and by the Biomedical Research Ethics Review Board of Inha University School of Medicine, Republic of Korea (INHA 15-013). Written consent was obtained from each individual prior to blood collection.

\section{Amplification and sequencing analysis of the PvLDH and PfLDH}

The genomic DNA of parasite was extracted from $200 \mu \mathrm{l}$ of whole blood using the QIAamp DNA Blood Kit (Qiagen, Hilden, Germany) following the manufacturer's instructions. PvLDH and PfLDH were amplified using PCR with primer sets described previous [15]. Briefly, the forward and reverse primers used to amplify PvLDH were $5^{\prime}$-ATGACGCCGAAACCCAAAAT- $3^{\prime}$ and $5^{\prime}$-ACCTTTAAATGAGCGCCTTCAT- ${ }^{\prime}$. The primers for PfLDH amplification was $5^{\prime}$-AGATGGCACCAA AAGCAAAAAT- $3^{\prime}$ and 5'-ACCTTTAAGCTAATG CCTTCAT- $3^{\prime}$. The thermal cycling parameters for the PCR were as follows: denaturation at $94{ }^{\circ} \mathrm{C}$ for $5 \mathrm{~min} ; 30$ cycles of $94{ }^{\circ} \mathrm{C}$ for $1 \mathrm{~min}$, annealing at $56{ }^{\circ} \mathrm{C}$ for $1 \mathrm{~min}$, and extension at $72{ }^{\circ} \mathrm{C}$ for $1 \mathrm{~min}$; and a final extension at $72{ }^{\circ} \mathrm{C}$ for $10 \mathrm{~min}$. To minimize nucleotide mismatches during amplification, Ex Taq DNA polymerase (Takara, Otsu, Japan), which has proofreading activity, was used in all PCR procedures. Each PCR product was analysed by electrophoresis on $1 \%$ agarose gels. The resulting PCR product was purified from the gel and cloned into the T\&A cloning vector (Real Biotech Corporation, Banqiao City, Taiwan). Each ligation mixture was transformed into Escherichia coli DH5 $\alpha$ competent cells and positive clones with the appropriate insert were screened by colony PCR. The nucleotide sequences of the cloned PCR product were analysed by automatic sequencing using the BigDye ${ }^{\mathrm{TM}}$ Terminator Cycle Sequencing Kit (Applied Biosystems, Foster City, CA, USA) on an ABI3730XL 
DNA analyzer (Applied Biosystems) with M13 forward and reverse primers. At least two clones from each isolate were sequenced to ensure sequencing accuracy, and some isolates underwent three or fourfold sequence coverage to confirm the existence of rare polymorphisms. The nucleotide sequences reported in this study have been deposited in the GenBank database under the accession numbers KU869730-KU869766 and KX885908KX885922 for PfLDH and KU895512-KU895548 and KX885923-KX885934 for PvLDH.

\section{Nucleotide sequence polymorphism analysis and neutrality test}

The nucleotide and deduced amino acid sequences of Myanmar PfLDH and PvLDH were analysed using EditSeq and SeqMan programs in the DNASTAR software package (DNASTAR, Madison, WI, USA). Nucleotide sequence polymorphism analysis was conducted for the $52 \mathrm{PfLDH}$ and $49 \mathrm{PvLDH}$ sequences. The number of segregating sites $(\mathrm{S})$, the number of haplotypes $(\mathrm{H})$, haplotype diversity $(\mathrm{Hd})$, nucleotide diversity $(\pi)$, and the average number of pairwise nucleotide differences within the population $(K)$ were estimated using DnaSP ver. 5.10.00. [20]. The $\pi$ was calculated to estimate the stepwise diversity throughout Myanmar PfLDH and PvLDH based on a sliding window of 50 base pairs (bp) with a step size of $10 \mathrm{bp}$. The values of synonymous (dS) and non-synonymous $(\mathrm{dN})$ substitutions were estimated and were compared using the Z-test $(P<0.05)$ in the MEGA4 program [21] using Nei and Gojobori's method [22] with the Jukes and Cantor correction. The Tajima's D value [23] and Fu and Li's D and F statistics [24] were analysed using the DnaSP ver. 5.10.00 to evaluate the neutral theory of evolution [20]. Sliding window plot analysis was also performed to analyse the step-wide value of Tajima's D throughout the Myanmar PfLDH and PvLDH based on a sliding window of $100 \mathrm{bp}$ with a step size of $25 \mathrm{bp}$. The recombination parameter $(\mathrm{R})$, which included the effective population size and probability of recombination between adjacent nucleotides per generation, and the minimum number of recombination events $(\mathrm{Rm})$ were determined using DnaSP ver. 5.10.00 [20].

\section{Genetic diversity of PvLDH and PfLDH in global Plasmodium falciparum and Plasmodium vivax populations} The genetic diversity of global PfLDH and PvLDH were analysed. The publicly available sequences of global PvLDH and PfLDH were used in this study (Additional files 1 and 2: Tables S1 and S2). Nucleotide sequence polymorphism analysis and a neutrality test for global $\operatorname{PvLDH}(n=100)$ and PfLDH $(n=334)$ population were estimated using DnaSP ver. 5.10.00 [20] and MEGA4 program [21] as described above. To investigate the genetic relationships among global PfLDH or PvLDH haplotypes, the haplotype network for global PfLDH $(n=334)$ and global PvLDH $(n=100)$ was separately analysed using NETWORK ver. 5.0.0.3 with the Median Joining algorithm [25].

\section{Homology modelling and mutant analysis of PvLDH and PfLDH}

The structural effects caused by amino acid changes identified in global PfLDH and PvLDH were analysed. The default protocol in the YASARA Structure ver.18.4.24 [26] was applied to construct three-dimensional (3D) homology models of PvLDH and PfLDH using the 'hm build.mcr' macro file (http://www.yasara.org/hm_build .mcr). To construct homology models, PSI-BLAST [27] was conducted against PDB entries [28]. The homology models were built based on each template and then were refined using high-resolution energy minimization of the YASARA force field [29]. The best homology model based on the PfLDH template (PDB code: 1T2D) [30] was chosen among the reliable models. The characteristics of the model were evaluated using a Ramachandran plot [31], ERRAT [32] and ProSA [33]. The crystal structure of the PfLDH model (PDB code: 1T2D) [30] was retrieved from the RCSB Protein Data Bank [34]. To determine the impact of mutations on the structural stability, thermodynamic changes in wild-type and mutant structures of PvLDH and PfLDH were predicted using SDM ver. 2 [35] by estimating a stability score. All mutant models were generated using Andante [36] as implemented in SDM ver. 2. CUPSAT [37] and MAESTROweb [38] and were also used to improve the overall prediction accuracy of mutations under consideration. All structural graphics were visualized using an open-source code of PyMOL (The PyMOL Molecular Graphics System, ver. 1.7.2.1, Schrödinger, LLC) in Linux. Structural superpositions onto the wild-type structure were performed and visualized using the 'cealign' method script (https://pymolwiki. org/index.php/Cealign) in PyMOL v1.7.2.1.

\section{Results}

\section{Genetic polymorphisms of Myanmar PvLDH}

A total of $49 \mathrm{PvLDH}$ sequences were obtained from Myanmar P. vivax isolates. All of the sequences contained 951 nucleotides encoding 316 amino acids, indicating no insertion or deletion existed in the analysed sequences. When the sequences were compared with Sal I PvLDH (GenBank ID: XM_001615570), 45 single nucleotide polymorphisms (SNPs) were identified at 29 positions. Thirty-one of the SNPs were synonymous, and the other 14 were non-synonymous. The non-synonymous SNPs resulted in amino acid changes at 13 positions in Myanmar PvLDH, including 12 
di-morphic amino acid changes (A20T, V37A, T62A, Y67C, V76A, V133A, G180D, K185R, Y250H, I273T, T277I, and T300A) and 1 tri-morphic amino acid change $(\mathrm{K} 301 \mathrm{M} / \mathrm{E})$. Based on these amino acid polymorphic patterns, Myanmar PvLDH was classified into 13 distinct haplotypes (haplotype 1 to 13) (Fig. 1a).
Genetic polymorphisms of Myanmar PfLDH

Fifty-two Myanmar PfLDH sequences were obtained. Comparing those sequences with the reference 3D7 PfLDH sequence (GenBank ID: XM_001349953) revealed 18 SNPs at 17 positions. Of these, 12 SNPs were non-synonymous and resulted in di-morphic amino acid changes at 11 positions (Fig. 1b). No insertion or deletion mutations were found in the Myanmar PfLDH sequences. Myanmar PfLDH was classified into

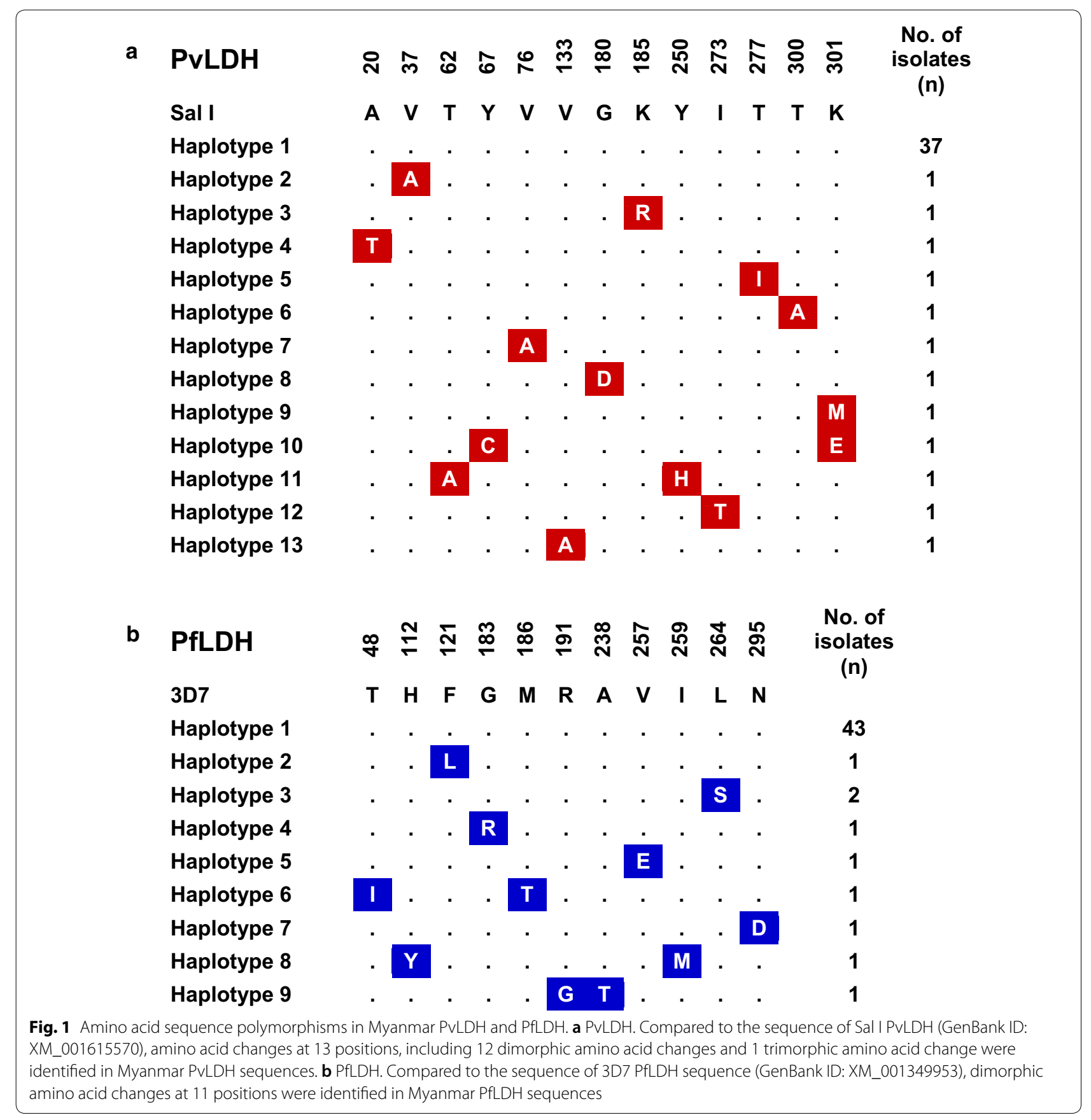




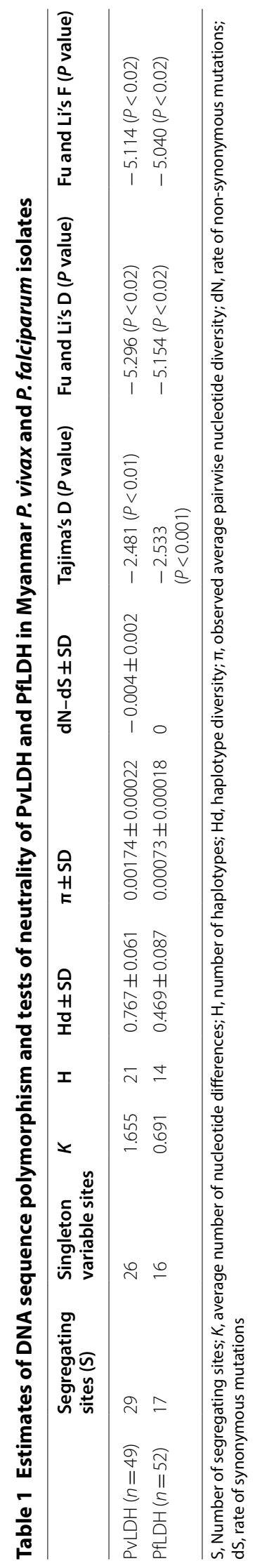


9 different haplotypes (haplotype 1 to 9 ) based on these amino acid polymorphic patterns (Fig. 1b).

\section{Genetic diversity and neutrality of Myanmar PvLDH and PfLDH}

Sequence analysis was performed to examine nucleotide diversity and neutrality of PvLDH and PfLDH among Myanmar $P$. vivax and $P$. falciparum population. The average number of pairwise nucleotide difference (K) for Myanmar PvLDH was 1.655 (Table 1). The overall haplotype diversity $(\mathrm{Hd})$ and nucleotide diversity $(\pi)$ of Myanmar PvLDH were estimated to be $0.767 \pm 0.061$ and $0.00174 \pm 0.00022$, respectively. Myanmar PvLDH was under negative selection as predicted by the negative values of Tajima's D $(-2.481, P<0.01)$, Fu and Li's $\mathrm{D}(-5.296, P<0.02)$ and Fu and Li's F $(-5.114, P<0.02)$. The $\mathrm{dN}-\mathrm{dS}$ value was $-0.004 \pm 0.002$. In the case of Myanmar PfLDH, the $K$ and Hd values were 0.691 and $0.469 \pm 0.087$, respectively (Table 1 ). The $\pi$ value was $0.00073 \pm 0.00018$. Myanmar PfLDH was also predicted to be under negative selection, which was supported by the negative values of Tajima's $\mathrm{D}(-2.533, P<0.001), \mathrm{Fu}$ and Li's D $(-5.154, P<0.02)$ and Fu and Li's F $(-5.040$,
$P<0.02)$. The value of $\mathrm{dN}-\mathrm{dS}$ was 0 . These results suggest that both PvLDH and PfLDH were under negative purifying selections in Myanmar $P$. vivax and $P$. falciparum populations. To examine whether specific region(s) of each LDH region were under selection, a sliding window plot analysis was performed. Nucleotide diversity $(\pi)$ was not evenly distributed throughout PvLDH and PfLDH of Myanmar $P$. vivax and $P$. falciparum populations. Higher nucleotide diversity was found in the $\mathrm{C}$-terminal regions of both PvLDH and PfLDH (Fig. 2). Sliding window analysis of Tajima's D values also showed similar patterns in PvLDH and PfLDH.

\section{Genetic polymorphism of PvLDH in the global P. vivax population}

Amino acid polymorphism patterns in global PvLDH $(n=100)$ were analysed. Compared to Sal I PvLDH (GenBank ID: XM_001615570), a total of 39 amino acid changes at 36 positions were identified in the global PvLDH population (Fig. 3). However, these amino acid changes were not commonly identified in global PvLDH, rather they differed by country. The amino acid changes found in Myanmar PvLDH were not identified
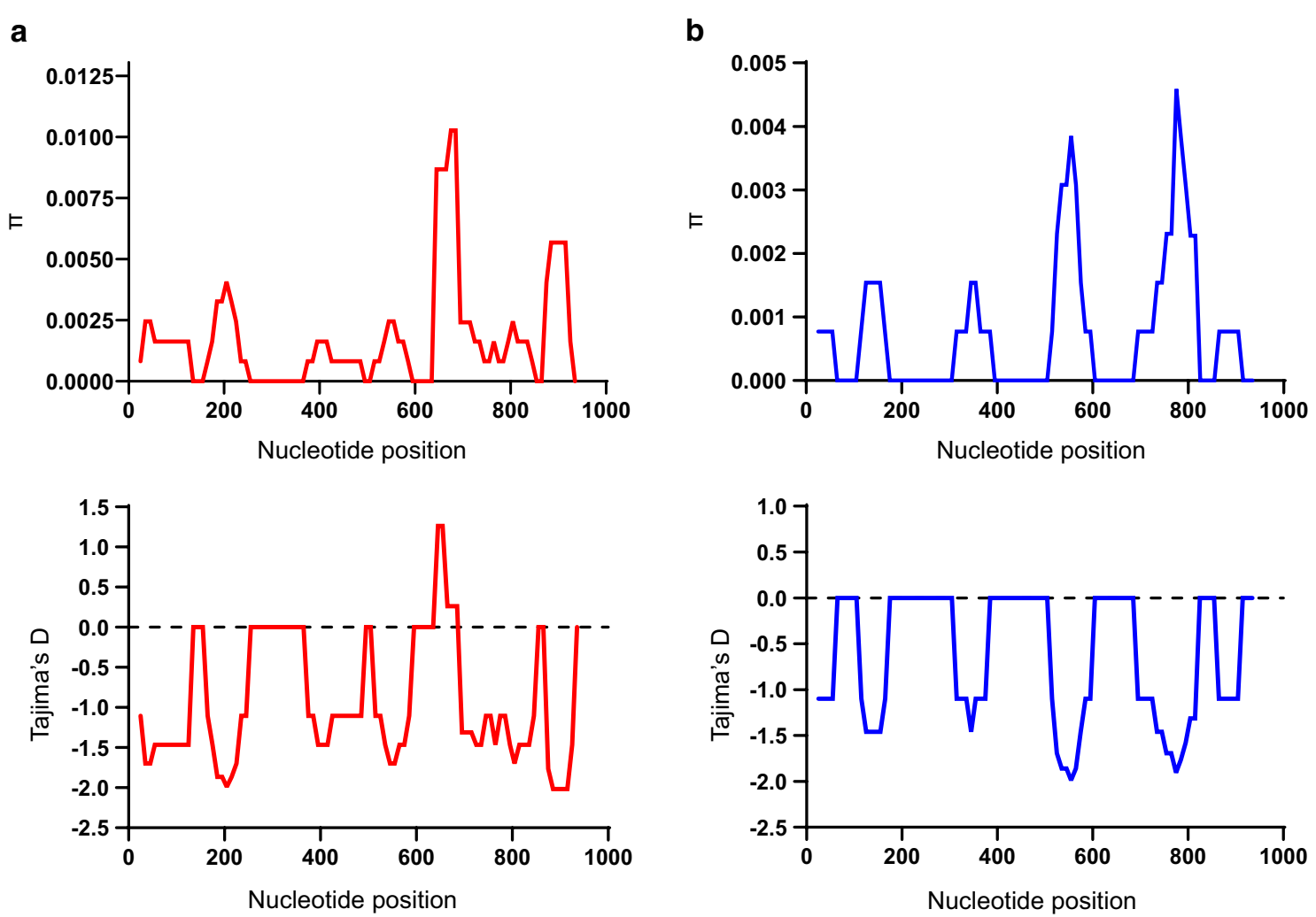

Fig. 2 Nucleotide diversity and natural selection of Myanmar PvLDH and PfLDH. Sliding window plot analyses show nucleotide diversity ( $\pi$ ) and Tajima's D values across the Myanmar PVLDH (a) and PfLDH (b) sequences analysed in this study. A window size of $100 \mathrm{bp}$ and a step size of $25 \mathrm{bp}$ were used 


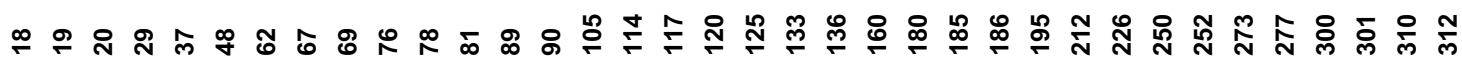

Sal I

$\begin{array}{lllllllllllllllllllllllllllllllllllll}V & M & A & G & V & T & T & Y & D & V & V & G & S & D & I & K & C & A & V & V & L & K & G & K & M & V & E & L & Y & K & I & T & T & K & K & M\end{array}$

Myanmar

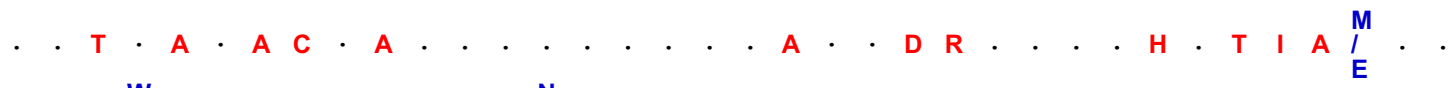

India

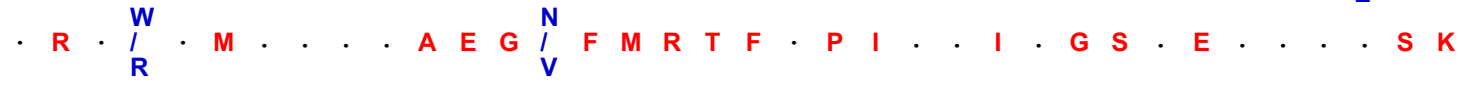

Iran

$\mathrm{L} \cdot \cdot \cdot$.

Korea

Madagascar

Fig. 3 Amino acid sequence polymorphisms in global PVLDH. A total of 100 global PVLDH sequences were compared to the Sal I PVLDH reference sequence (GenBank ID: XM_001615570). A total of 36 amino acid changes, including 33 di-morphic amino acid changes and 3 tri-morphic amino acid changes, were identified among global PVLDH sequences

in PvLDH sequences from other countries including India, Iran, Korea and Madagascar. Similarly, amino acid changes found in other countries were country-specific. Most amino acid polymorphisms were di-morphic except for three amino acid changes that were tri-morphic (K301M/E in Myanmar PvLDH and G29W/R and D90N/V in India PvLDH). All the amino acid changes were observed at low frequencies ranging from 1 to $2 \%$ in the global PvLDH population.

\section{Genetic polymorphism of PfLDH in the global P. falciparum population}

Amino acid polymorphism patterns in global PfLDH $(n=334)$ were analysed. Compared to 3D7 PfLDH (GenBank ID: XM_001349953), a total of 19 amino acid polymorphisms, all di-morphic, were identified at 19 positions (Fig. 4). Similar to global PvLDH, global PfLDH showed different patterns of amino acid changes by country. Most amino acid changes (17 of 19) were identified in Myanmar and China PfLDH. Only one amino acid change (D272N) was commonly observed in isolates from several countries including India, Iran, Gambia, Madagascar, Senegal and Uganda PfLDH. No amino acid change was observed in PfLDH from Gabon, Ghana, Kenya, Sudan, Republic of Congo, Mali, Honduras, and Brazil. The overall frequencies of the amino acid substitutions in the global PfLDH population were low and ranged from 0.3 to $3.3 \%$.

\section{Genetic diversity and neutrality of global PvLDH and PfLDH}

To further examine whether natural selection had contributed to PvLDH and PfLDH diversity within the global $P$. vivax and $P$. falciparum populations, publicly available sequences deposited in the NCBI and PlasmoDB databases were analysed (Table 2). The $\mathrm{dN}-\mathrm{dS}$ values for global PvLDH and PfLDH were 0 and $-0.004 \pm 0.002$, respectively, indicating both genes were under strict purifying selections. In order to more closely explore the effects of natural selection on each PvLDH and PfLDH, the values for Tajima's D, Fu and Li's D and Fu and Li's F were analysed. The Tajima's D values for global PvLDH and PfLDH were $-2.26226(P<0.01)$ and -2.28117 $(P<0.01)$, respectively. The values for Fu and Li's $\mathrm{D}$ and $\mathrm{Fu}$ and Li's $\mathrm{F}$ for global PvLDH and PfLDH were also negative, indicating both genes had been influenced by negative natural selection (Table 2). Sliding window plot analyses of nucleotide diversity $(\pi)$ and Tajima's D of the global PvLDH and PfLDH also revealed low genetic diversity and negative selection (Fig. 5). A haplotype network analysis of global PvLDH $(n=100)$ showed 35 closely linked haplotypes (Fig. 6). Two major haplotypes ( $\mathrm{H} 1$ and $\mathrm{H} 8)$ were identified, and the other haplotypes were singletons arising from $\mathrm{H} 1$ to $\mathrm{H} 8$. The most prevalent haplotype was haplotype 1 (H1), which shared the same nucleotide sequences as Sal I (GenBank ID: XM_001615570) with a frequency of $38.0 \%$. The frequency of haplotype $8(\mathrm{H} 8)$ was $29.0 \%$. A haplotype network analysis of global PfLDH $(n=334)$ revealed a total of 22 distinct haplotypes (Fig. 7). The most prevalent haplotype was $\mathrm{H} 4$, which had the identical nucleotide sequences with 3D7 (GenBank ID: XM_001349953) and was shared by populations from different countries with a frequency of $83.2 \%$. Haplotype 5 (H5) was the second most prevalent haplotype (frequency of 10.4\%) and was mainly comprised of African PfLDH. Nineteen haplotypes, except for haplotype 6 (H6), were singletons. These haplotypes were linked to the most frequent $\mathrm{H} 4$ by short or long branches. Similar to global PvLDH, haplotype network analysis of global PfLDH revealed a simple 


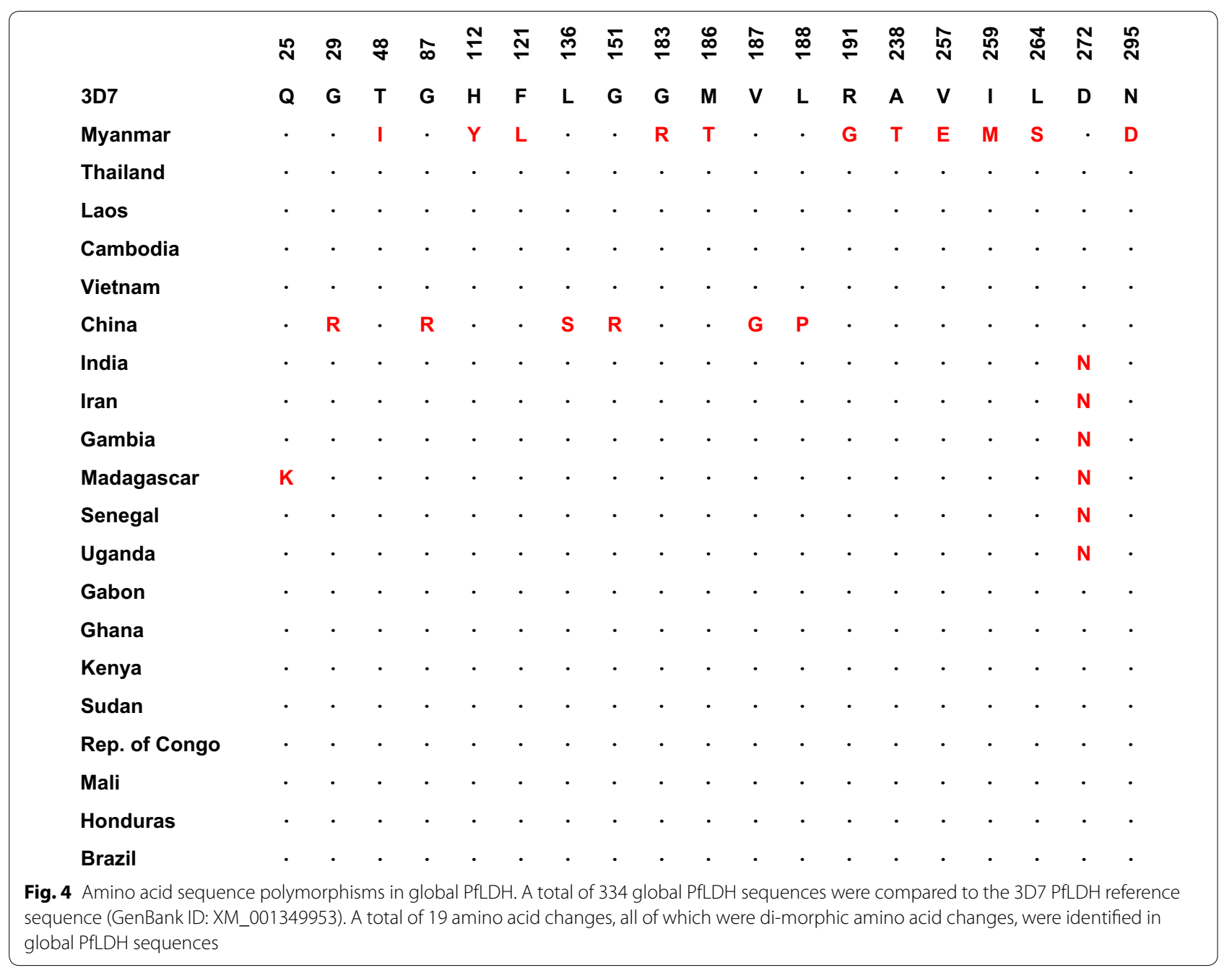

cluster with a small number of haplotypes having low frequencies separated by short branches.

\section{Structural impacts caused by amino acid changes in global isolates of PvLDH and PfLDH}

The structural effects of amino acid changes found in global isolates of PvLDH and PfLDH were investigated. Several PvLDH crystal structures (PDB codes: 5HRU at $1.71 \AA, 5 \mathrm{HTO}$ at $1.90 \AA$ and $2 \mathrm{~A} 92$ at $2.04 \AA$ ) were reported previously (Additional file 3: Table S3), but their resolutions were not high, Accuracy of the template structure such as resolution should be carefully considered to construct the most reliable homology model [39]. Therefore, a homology model of PvLDH was constructed based on a high-resolution crystal structure of PfLDH (PDB code: 1 T2D at $1.1 \AA$ resolution), due to the low-resolution crystal structures for PvLDH. The constructed PvLDH model had 94.1\% of the amino acid residues in a favorable region according to the Ramachandran plot (Additional file 4: Fig. S1a), a ProSA Z-score of - 9.82 (Additional file 4: Fig. S1b) and an ERRAT overall quality score of 96.74 (Additional file 4: Fig. S1c). Superposition between PvLDH and PfLDH showed a root-mean-square deviation (RMSD) of 0.06, suggesting the two proteins shared the same overall folding pattern (Additional file 5: Fig. S2a). The models predicted a Rossmann fold in all pLDH enzymes and the NADH cofactor-binding site $[40,41]$ that forms a series of alternating $6 \beta$-strands and $5 \alpha$-helices (Additional file 5: Fig. S2b). Furthermore, PvLDH and PfLDH shared identical amino acid sequences at catalytic residues (R95, D155, R158, and H182) [42], the active site (K84), cofactor binding sites (P235 and P239) [43], and the substrate-specific loop (DKEWN, amino acid positions 90-94) [44] (Additional file 5: Fig. S2a). When all the amino acid changes found in global PvLDH and PfLDH were applied to the models, it suggested that they were scattered throughout the structures (Figs. 8 


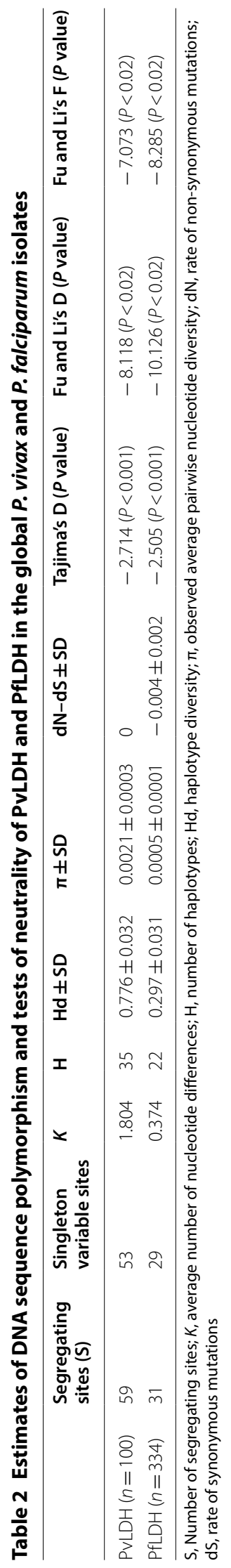


a
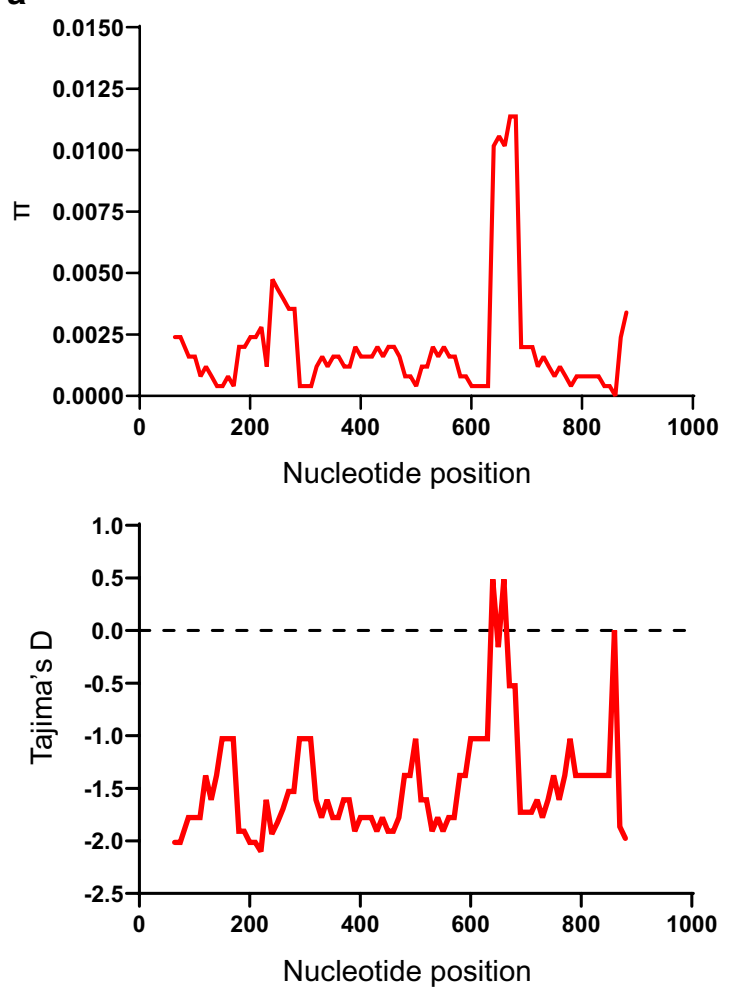

b
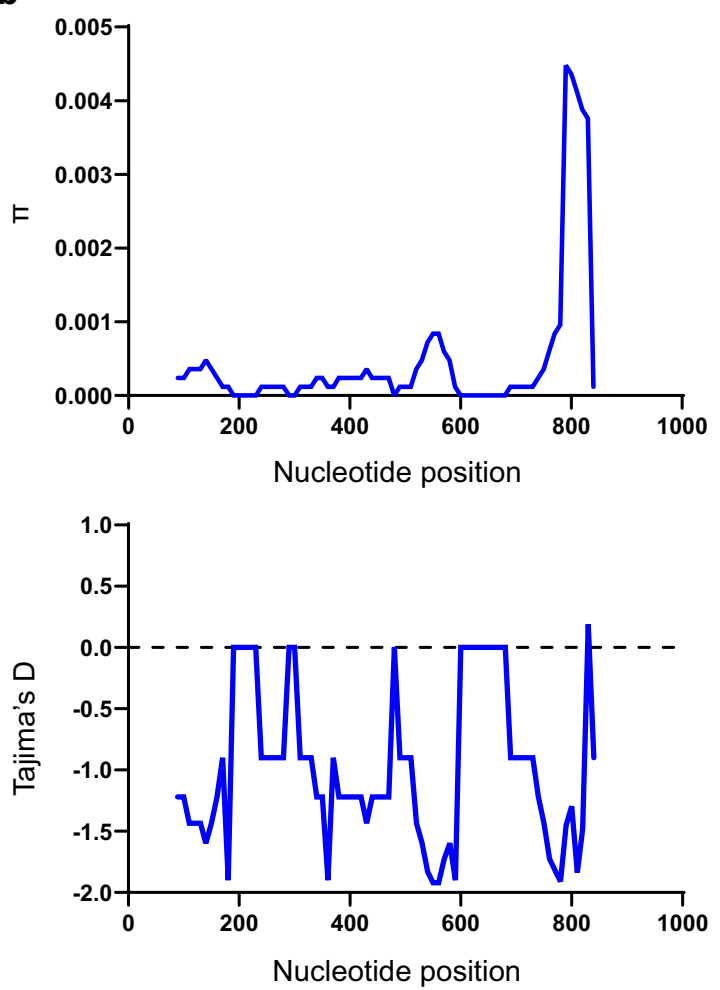

Fig. 5 Nucleotide diversity and natural selection of global PVLDH and PfLDH. a PVLDH. Sliding window plot analyses show nucleotide diversity ( $\pi$ ) and Tajima's D values across global PVLDH sequences. A window size of $100 \mathrm{bp}$ and a step size of $25 \mathrm{bp}$ were used. b PfLDH. Sliding window plot analyses show nucleotide diversity $(\pi)$ and Tajima's D values across global PfLDH sequences. A window size of 100 bp and a step size of 25 bp were used

and 9). The structural impact of all non-synonymous amino acid substitutions of PvLDH and PfLDH were analysed based on the RMSD and the predicted $\Delta \Delta G$ $(\mathrm{kcal} / \mathrm{mol})$ values between the wild-type and mutants.

Superposition between PvLDH wild-type and 40 mutants generated an RMSD of 0.001-0.059 (Additional file 6: Table S4). PfLDH wild-type and 19 mutants generated an RMSD of 0.001-0.053 (Additional file 7: Table S5). These results suggest that individual amino acid changes may not induce significant structural changes in both PvLDH and PfLDH. To improve the accuracy of potential stability changes $(\Delta \Delta \mathrm{G}, \mathrm{kcal} / \mathrm{mol})$ with each amino acid change, three different predictors (SDM ver. 2 [35], CUPSAT [37], and MAESTROweb [38]) were applied, but their coincidence results were inconclusive (Additional files 6 and 7: Tables S4 and S5). Although D90V/N of PvLDH was located in the substrate-specific loop, it is predicted not to significantly affect structural stability (Additional file 6: Table S4). These results collectively suggest that all amino acid changes identified in global PvLDH and PfLDH are unlikely to cause significant structural changes in the proteins.

\section{Discussion}

Analyses of genetic polymorphisms in Myanmar PvLDH and PfLDH revealed a low level of genetic diversity in Myanmar P. vivax and P. falciparum populations. Both genes showed synonymous SNPs and non-synonymous SNPs, which generated different haplotypes in each gene: 13 haplotypes in PvLDH and 9 haplotypes in PfLDH. Global PvLDH and PfLDH sequence analyses also suggested that amino acid changes caused by diverse nonsynonymous SNPs were identified among the global PvLDH and PfLDH. However, the frequencies of the amino acid changes were not high, and they were not evenly distributed among the global population but were country-specific. Overall genetic diversity was greater in PvLDH than PfLDH. This is consistent with previous findings on the genetic diversity of PvLDH and PfLDH in other countries [15-17, 42, 45]. These results are also consistent with a previous report of comparative genomic analyses suggesting more polymorphic events were identified in the P. vivax genome than P. falciparum [46].

Neutrality tests of Myanmar PvLDH and PfLDH indicated the influence of a strong purifying selection on 


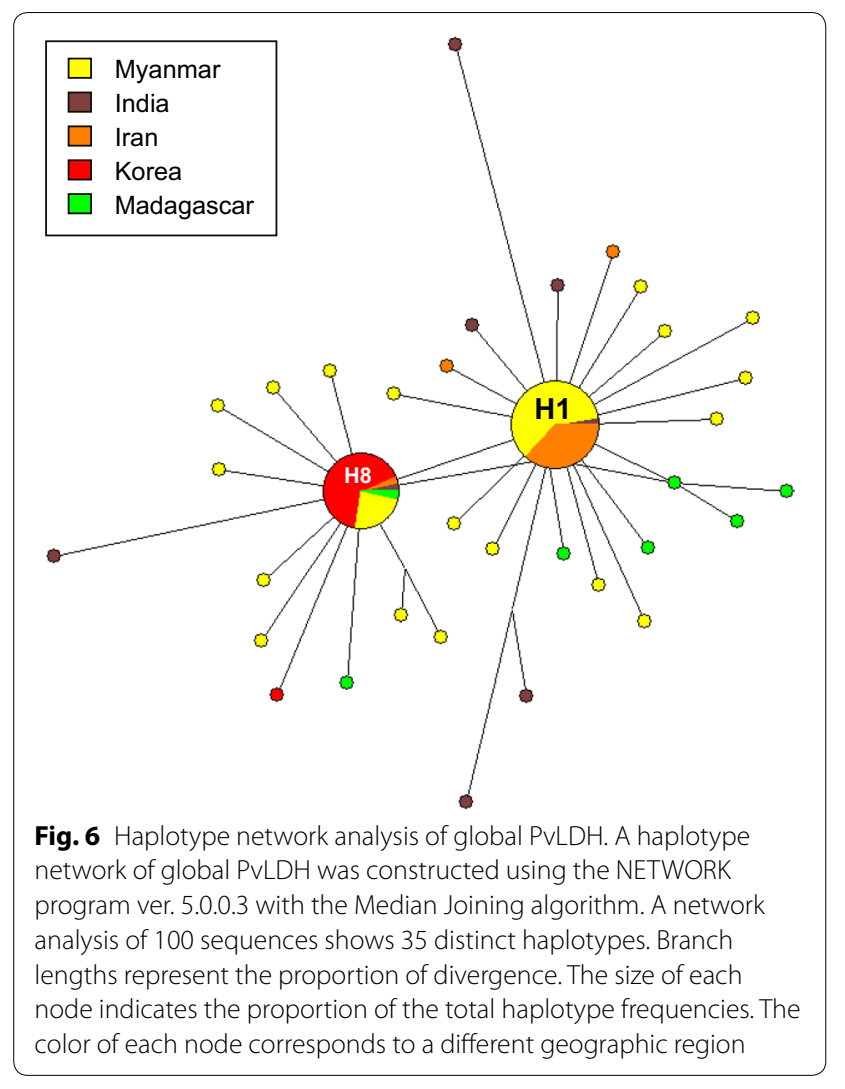

Myanmar PvLDH and PfLDH. The $\mathrm{dN}-\mathrm{dS}$ values of Myanmar PvLDH and PfLDH did not show a significant departure from zero. The significant negative values of Tajima's D, Fu and Li's D, and Fu and Li's F for both PvLDH and PfLDH were also in agreement with the $\mathrm{dN}-$ dS values. Similar patterns of purifying selection were also identified in global PvLDH and PfLDH [17], suggesting the genetic conservation of global PvLDH and PfLDH was mostly maintained by purifying selection. This is probably due to functional constraints. Haplotype network analyses of global PvLDH and PfLDH were also consistent with purifying selection, each with one or a few modal haplotypes separated by short branches.

A total of 39 amino acid changes at 36 positions were identified in the 100 global PvLDH sequences analysed in this study. Regarding PfLDH, 19 amino acid changes at 19 positions were observed in the 334 global sequences. The amino acid changes found in global PvLDH and PfLDH were not all located at essential residues, including catalytic residues (R95, D155, R158 and H182), the active site (K84), cofactor binding sites (P235 and P239) and the substrate-specific loop (DKEWN, amino acid positions 90-94) [47]. One exception to this is $\mathrm{D} 90 \mathrm{~V} / \mathrm{N}$, which was found in the substrate-specific loop of PvLDH. The mutation $\mathrm{D} 90 \mathrm{~V} / \mathrm{N}$, however, does not alter the protein's

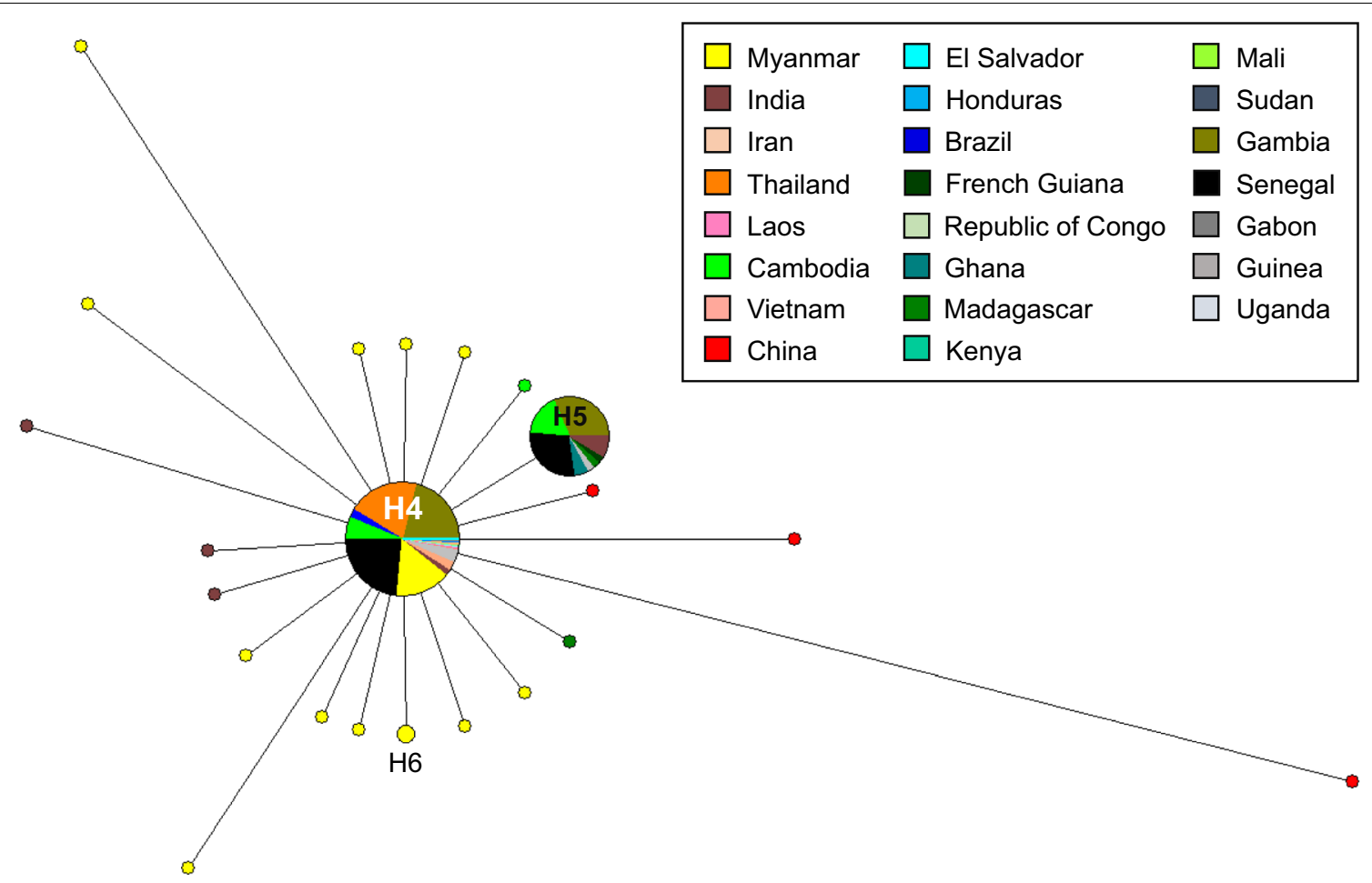

Fig. 7 Haplotype network analysis of global PfLDH. Haplotype network of global PfLDH was constructed using the NETWORK program ver. 5.0.0.3 with the Median Joining algorithm. A network analysis of 334 sequences shows 22 individual haplotypes. Branch lengths represent the proportion of divergence. The size of each node indicates the proportion of the total haplotype frequencies. The color of each node corresponds to a different geographic region 


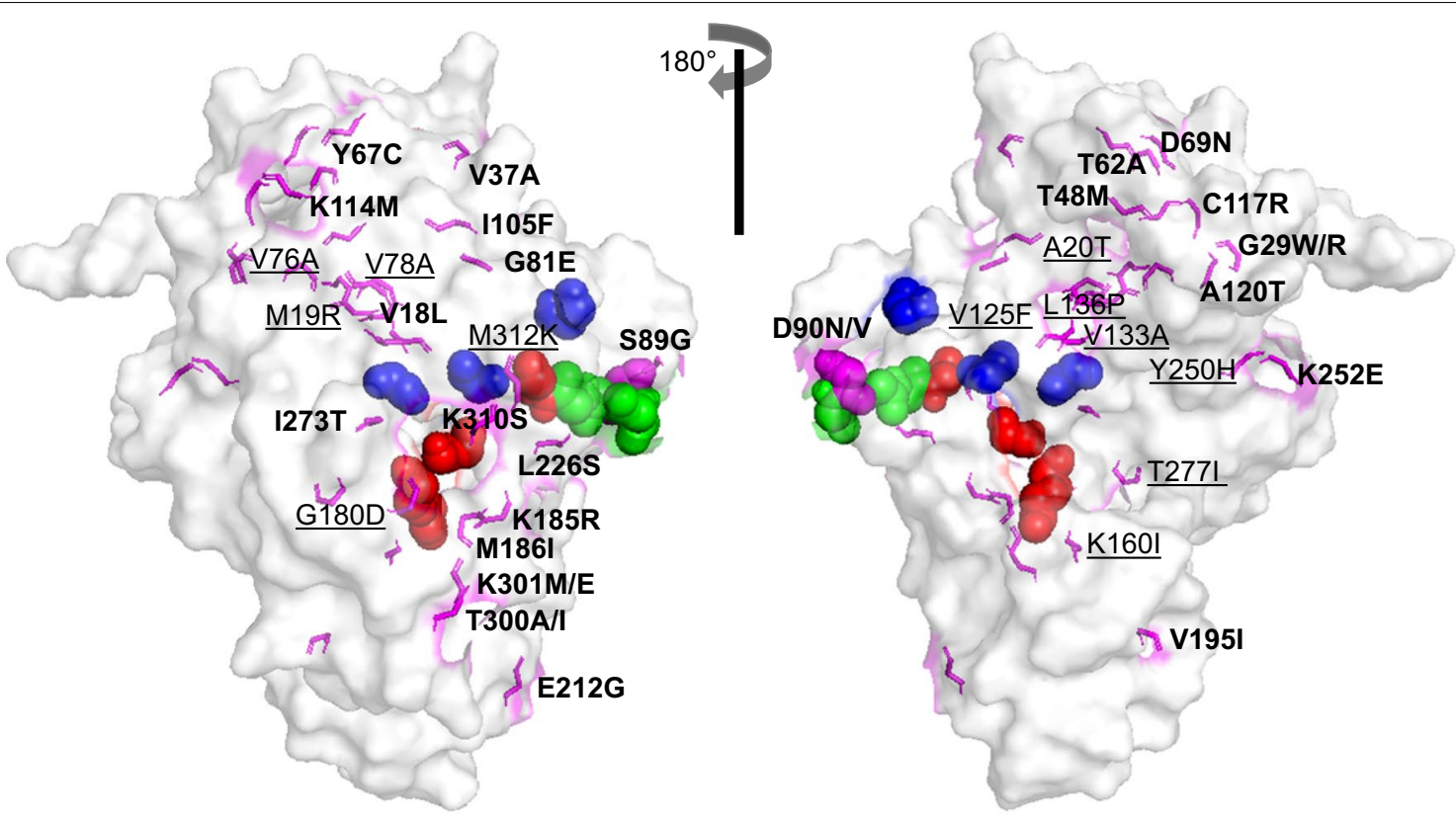

Fig. 8 Polymorphism sites mapped to the 3D structure of PvLDH. Front and back views of the surface of PVLDH with mutated amino acid residues. The two views are rotated with respect to each other by $180^{\circ}$ around the vertical axis. The 36 amino acid changes identified in global PvLDH are indicated with magenta sticks. The mutated residues, which were located on the external or internal regions, are depicted in bold or underlined, respectively. The functional residues of PVLDH are depicted as colored spheres: catalytic residues (R95, D155, R158 and H182) in red, the active site (K84) and cofactor-binding site (P235 and P239) in blue, and the substrate-specific loop (D90-N94) in green

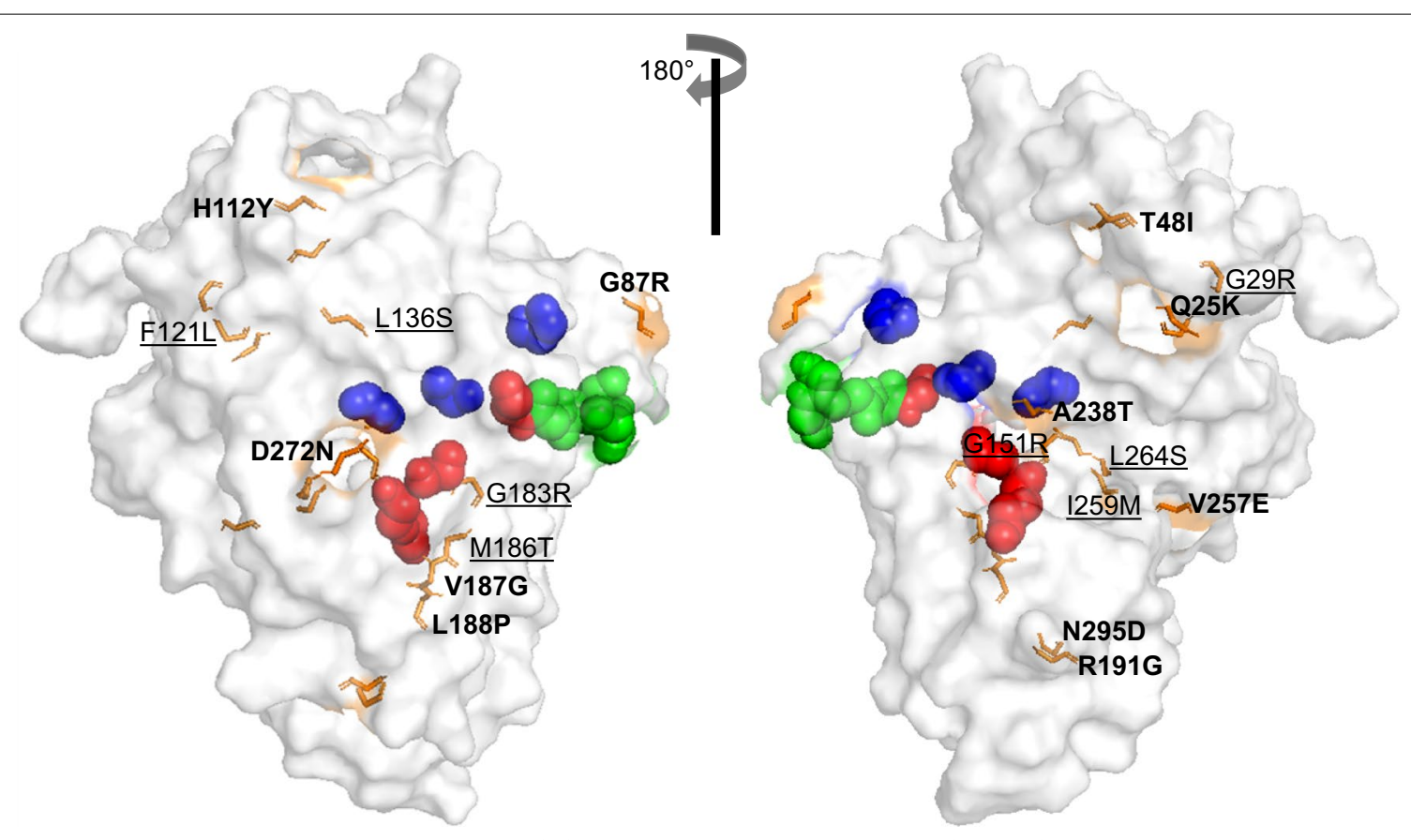

Fig. 9 Polymorphism sites mapped to the 3D structure of PfLDH. Front and back views of the surface of PfLDH with mutated amino acid residues. The two views are rotated with respect to each other by $180^{\circ}$ around the vertical axis. The 19 amino acid changes identified in global PfLDH are indicated with orange sticks. The mutation residues, which were located on the external or internal regions, were depicted in bold or underlined, respectively. The functional residues of PfLDH sre depicted as colored spheres: catalytic residues (R95, D155, R158 and H182) in red, the active site (K84) and cofactor-binding site (P235 and P239) in blue, and the substrate-specific loop (D90-N94) in green 
structural stability since no prediction demonstrated a significant impact on protein stability. These results suggest that the amino acid changes in global PvLDH and PfLDH are unlikely to cause significant structural change that may disrupt the function of the enzymes. The 3D structure analyses also supported this notion by suggesting that no significant conformational change is likely to be caused by these amino acid variations.

Concerns have been raised about the genetic diversity found in pLDH and how it might influence the diagnostic efficacy of pLDH-based RDTs [48-50]. However, the results of this study suggest that the two genes were well-conserved in global populations even though low frequencies of amino acid changes were identified in the global population. It is unlikely that these polymorphic patterns identified in global PvLDH and PfLDH can influence the diagnostic performance of pLDHbased RDTs. In fact, pLDH-based RDTs performed well in filed performance assessments in India and other countries despite the occurrence of different genotypes [51-53]. Blood samples used in this study were also correctly diagnosed to each species by pLDH-based RDT kits (unpublished observations). These collectively suggest that epitopes of PvLDH and PfLDH targeted in RDTs are well-conserved in the global populations and therefore pLDH-based RDTs would be reliable to diagnose malaria in the field conditions. However, it would be noteworthy to mention that accuracy of RTDs can be affected by low parasitaemia and residual parasite antigens after clearance $[4,6,54]$. And therefore, continuous efforts to develop RDTs with higher sensitivity and specificity should be performed.

Functional and structural constraints of global PvLDH and PfLDH are also highly informative since pLDH is a promising drug target [55]. Plasmodial LDH is an essential enzyme in the central metabolic pathway of malaria parasites $[14,56-58]$. Due to substantial differences in the human ortholog, pLDH is an attractive target for developing anti-malarial drugs $[47,59]$. Inhibition of pLDH prevents adenosine triphosphate (ATP) production, which kills malaria parasites [60]. The results obtained in this study may help to identify or design effective inhibitors for anti-malarial drugs targeting pLDH.

The limitation of this study is that only limited numbers of Myanmar and global PvLDH and PfLDH sequences were included in the analysis. To better understand the polymorphic nature of PvLDH and $\mathrm{PfLDH}$, further analysis of PvLDH and PfLDH variations in the global $P$. vivax and $P$. falciparum populations would be necessary.

\section{Conclusions}

Low levels of genetic diversity, which may be affected by strong purifying selection, were identified in global PvLDH and PfLDH populations. Although non-synonymous SNPs that induce amino acid changes in global PvLDH and PfLDH were found, these amino acid changes were not commonly distributed in the global population, and their frequencies were low. Despite differences in the amino acid sequence, functionally important residues that maintain the structure and function of pLDH are well-conserved. These data collectively suggest that $\mathrm{pLDH}$ is a useful target molecule for RDT. Further examination of the genetic diversity of pLDH in diverse global $P$. vivax and $P$. falciparum populations with a larger number of isolates is necessary to better understand the polymorphic nature and evolutionary aspects of PvLDH and PfLDH.

\section{Supplementary information}

Supplementary information accompanies this paper at https://doi. org/10.1186/s12936-020-3134-y.

\begin{abstract}
Additional file 1: Table S1. Accession numbers of global PfLDH sequences.

Additional file 2: Table S2. Accession numbers of global PVLDH sequences.

Additional file 3: Table S3. The homology modeling templates (Top 40 hits).

Additional file 4: Fig. S1. Quality validation of the PVLDH homology models. (a) Ramachandran plot showing that most amino acid residues the residues (94.1\%) are located in favored regions, allowed regions (5.1\%), generously allowed regions (0.4\%), and disallowed regions (0.4\%). Red (A, $B, L)$, yellow $(a, b, l, p)$ and light yellow $(\sim a, \sim b, \sim 1, \sim p)$ indicate the most favored regions, allowed regions, and generously allowed regions, respectively. White indicates disallowed regions. All non-glycine and non-proline residues are shown as closed black squares while glycines (non-end) are shown as closed black triangles. Disallowed residues are colored in red. (b) The ProSA energy profile [33] indicates a Z-score of - 9.82. (c) In the ERRAT plot [32], the overall quality factor is $96.74 \%$.

Additional file 5: Fig. S2. Structural comparison between PVLDH and PfLDH. (a) Semi-transparent cartoon representations of PVLDH in cyan and PfLDH in orange reveal a similar overall fold with 0.06 RMSD. All functional residues are identical to the amino acids in the corresponding positions; catalytic residues (R95, D155, R158 and H182) in red spheres, the active site (K84) and cofactor-binding site (P235 and P239) in blue spheres, and the substrate-specific loop (D90-N94) in green spheres. (b) Perpendicular view of (a) shows a Rossmann fold-like subdomain, which is composed of $6 \beta$-strands and 5 a-helices.
\end{abstract}

Additional file 6: Table S4. Potential changes of in the stability of PVLDH caused by 36 mutations using three different predictors.

Additional file 7: Table S5. Potential changes of in the stability of PfLDH caused by 19 mutations using three different predictors.

Abbreviations

$\mathrm{dN}$ : Rate of non-synonymous mutations; dS: Rate of synonymous mutations; $\mathrm{H}$ : Number of haplotypes; Hd: Haplotype diversity; K: Average number of nucleotide differences; LDH: Lactate dehydrogenase; PCR: Polymerase chain 
reaction; pLDH: Lactate dehydrogenase of Plasmodium spp; PvLDH: Lactate dehydrogenase of Plasmodium vivax; PfLDH: Lactate dehydrogenase of Plasmodium falciparum; $\pi$ : Observed average pairwise nucleotide diversity; rRNA: 18 S ribosomal RNA; RMSD: Root-mean-square deviation; S: Number of segregating sites.

\section{Acknowledgements}

We thank the staffs in the Department of Medical Research Pyin Oo Lwin Branch and the health professionals in Naung Cho and Pyin Oo Lwin townships for their contribution and technical support during blood collection.

\section{Authors' contributions}

$J L$ and TIK performed the genetic analysis of PvLDH and PfLDH. JL, SKA, MKM, and KL contributed to blood sample collection. JL, TIK, HGL, WGY, JMK, and BKN analysed and interpreted the data. TSK and BKN designed and supervised the experiments. JL, HGL,WGY, and BKN drafted the manuscript. All authors read and approved the final manuscript.

\section{Funding}

This research was supported by the National Research Foundation of Korea (NRF) grants funded by the Korean Government (NRF-2019K1A3A9A01000005) and the Ministry of Education (NRF-2017R1A6A3A11030620).

\section{Availability of data and materials}

The data supporting the conclusions of this article are provided within the article and its additional files. The original datasets analysed in this current study are available from the corresponding author upon request. The nucleotide sequences reported in this study have been deposited in the GenBank database under the accession numbers KU869730-KU869766 and KX885908KX885922 for PfLDH and KU895512-KU895548 and KX885923-KX885934 for PVLDH.

\section{Ethics approval and consent to participate}

This study was approved by the Ethics Review Committee, Department of Medical Research, Myanmar (97/Ethics 2015) and by the Ethical Review Committee of Inha University School of Medicine, Korea (INHA 15-013). Informed written consent and permission were obtained from each individual.

\section{Consent for publication}

Not applicable.

\section{Competing interests}

The authors declare that they have no competing interests.

\section{Author details}

1 Department of Tropical Medicine and Inha Research Institute for Medical Science, Inha University School of Medicine, Incheon, Republic of Korea.

${ }^{2}$ Department of Parasitology and Tropical Medicine, and Institute of Health Sciences, Gyeongsang National University College of Medicine, Jinju 52727, Republic of Korea. ${ }^{3}$ BK21Plus Team for Anti-aging Biotechnology and Industry, Department of Convergence Medical Science, Gyeongsang National University, Jinju 52727, Republic of Korea. ${ }^{4}$ Department of Medical Environmental Biology, Chung-Ang University College of Medicine, Seoul 06974, Republic of Korea. ${ }^{5}$ Department of Medical Research Pyin Oo Lwin Branch, Pyin Oo Lwin, Myanmar. ${ }^{6}$ Present Address: Planning and Management Division, Nakdonggang National Institute of Biological Resources, Sangju 37242, Republic of Korea.

Received: 28 November 2019 Accepted: 22 January 2020

Published online: 04 February 2020

\section{References}

1. WHO. World malaria report 2008. Geneva: World Health Organization; 2008. p. 1-190.

2. Okell LC, Ghani AC, Lyons E, Drakeley CJ. Submicroscopic infection in Plasmodium falciparum-endemic populations: a systematic review and meta-analysis. J Infect Dis. 2009;200:1509-17.

3. Payne D. Use and limitations of light microscopy for diagnosing malaria at the primary health care level. Bull World Health Organ. 1988;66:621-6.
4. Chong CK, Cho PY, Na BK, Ahn SK, Kim JS, Lee JS, et al. Evaluation of the accuracy of the easy test malaria Pf/Pan Ag, a rapid diagnostic test, in Uganda. Korean J Parasitol. 2014,52:501-5.

5. Moges B, Amare B, Belyhun Y, Tekeste Z, Gizachew M, Workineh M, et al. Comparison of CareStart HRP2/pLDH COMBO rapid malaria test with light microscopy in north-west Ethiopia. Malar J. 2012;11:234.

6. Nyunt MH, Kyaw MP, Win KK, Myint KM, Nyunt KM. Field evaluation of HRP2 and pan pLDH-based immunochromatographic assay in therapeutic monitoring of uncomplicated falciparum malaria in Myanmar. Malar J. 2013;12:123.

7. Gamboa D, Ho MF, Bendezu J, Torres K, Chiodini PL, Barnwell JW, et al. A large proportion of $P$. falciparum isolates in the Amazon region of Peru lack pfhrp2 and pfhrp3: implications for malaria rapid diagnostic tests. PLoS ONE. 2010;5:e8091.

8. Maltha J, Gamboa D, Bendezu J, Sanchez L, Cnops L, Gillet P, et al. Rapid diagnostic tests for malaria diagnosis in the Peruvian Amazon: impact of pfhrp2 gene deletions and cross-reactions. PLOS ONE. 2012;7:e43094.

9. Bendezu J, Rosas A, Grande T, Rodriguez H, Llanos-Cuentas A, Escobedo $J$, et al. Field evaluation of a rapid diagnostic test (Parascreen) for malaria diagnosis in the Peruvian Amazon. Malar J. 2010;9:154.

10. Kumar N, Pande V, Bhatt RM, Shah NK, Mishra N, Srivastava B, et al. Genetic deletion of HRP2 and HRP3 in Indian Plasmodium falciparum population and false negative malaria rapid diagnostic test. Acta Trop. 2013;125:119-21.

11. Li P, Xing H, Zhao Z, Yang Z, Cao Y, Li W, et al. Genetic diversity of Plasmodium falciparum histidine-rich protein 2 in the China-Myanmar border area. Acta Trop. 2015;152:26-31.

12. WHO. False-negative RDT results and implications of new reports of $P$. falciparum histidine-rich protein 2/3 gene deletions. Geneva: Global Malaria Programme; 2017. p. 1-11.

13. Brown WM, Yowell CA, Hoard A, Vander Jagt TA, Hunsaker LA, Deck LM, et al. Comparative structural analysis and kinetic properties of lactate dehydrogenases from the four species of human malarial parasites. Biochemistry. 2004;43:6219-29.

14. Shakespeare PG, Trigg PI, Kyd SI, Tappenden L. Glucose metabolism in the simian malaria parasite Plasmodium knowlesi: activities of the glycolytic and pentose phosphate pathways during the intraerythrocytic cycle. Ann Trop Med Parasitol. 1979;73:407-15.

15. Getacher Feleke D, Nateghpour M, Motevalli Haghi A, Hajjaran H, Farivar L, Mohebali M, et al. DNA sequence polymorphism of the lactate dehydrogenase gene from Iranian Plasmodium vivax and Plasmodium falciparum isolates. Iran J Parasitol. 2015;10:505-16.

16. Shin HI, Kim JY, Lee WJ, Sohn Y, Lee SW, Kang YJ, et al. Polymorphism of the parasite lactate dehydrogenase gene from Plasmodium vivax Korean isolates. Malar J. 2013;12:166.

17. Simpalipan P, Pattaradilokrat S, Harnyuttanakorn P. Global sequence diversity of the lactate dehydrogenase gene in Plasmodium falciparum. Malar J. 2018;17:16.

18. Kang JM, Cho PY, Moe M, Lee J, Jun H, Lee HW, et al. Comparison of the diagnostic performance of microscopic examination with nested polymerase chain reaction for optimum malaria diagnosis in Upper Myanmar. Malar J. 2017;16:119.

19. Snounou G, Singh B. Nested PCR analysis of Plasmodium parasites. Methods Mol Med. 2002;72:189-203.

20. Librado P, Rozas J. DnaSP v5: a software for comprehensive analysis of DNA polymorphism data. Bioinformatics. 2009;25:1451-2.

21. Tamura K, Dudley J, Nei M, Kumar S. MEGA4: Molecular evolutionary genetics analysis (MEGA) software version 4.0. Mol Biol Evol. 2007;24:1596-9.

22. Nei M, Gojobori T. Simple methods for estimating the numbers of synonymous and nonsynonymous nucleotide substitutions. Mol Biol Evol. 1986;3:418-26.

23. Tajima F. Statistical method for testing the neutral mutation hypothesis by DNA polymorphism. Genetics. 1989;123:585-95.

24. Fu YX, Li WH. Statistical tests of neutrality of mutations. Genetics. 1993;133:693-709.

25. Bandelt HJ, Forster $P$, Rohl A. Median-joining networks for inferring intraspecific phylogenies. Mol Biol Evol. 1999;16:37-48.

26. Krieger E, Nabuurs SB, Vriend G. Homology modeling. Methods Biochem Anal. 2003;44:509-23. 
27. Altschul SF, Madden TL, Schaffer AA, Zhang J, Zhang Z, Miller W, et al. Gapped BLAST and PSI-BLAST: a new generation of protein database search programs. Nucleic Acids Res. 1997;25:3389-402.

28. Bernstein FC, Koetzle TF, Williams GJ, Meyer EF Jr, Brice MD, Rodgers JR, et al. The Protein Data Bank. A computer-based archival file for macromolecular structures. Eur J Biochem. 1977;80:319-24.

29. Krieger E, Joo K, Lee J, Lee J, Raman S, Thompson J, et al. Improving physical realism, stereochemistry, and side-chain accuracy in homology modeling: four approaches that performed well in CASP8. Proteins. 2009;77(Suppl 9):114-22.

30. Cameron A, Read J, Tranter R, Winter VJ, Sessions RB, Brady RL, et al. Identification and activity of a series of azole-based compounds with lactate dehydrogenase-directed anti-malarial activity. J Biol Chem. 2004;279:31429-39.

31. Lovell SC, Davis IW, Arendall WB 3rd, de Bakker PI, Word JM, Prisant MG, et al. Structure validation by Calpha geometry: phi, psi and Cbeta deviation. Proteins. 2003;50:437-50.

32. Colovos C, Yeates TO. Verification of protein structures: patterns of nonbonded atomic interactions. Protein Sci. 1993;2:1511-9.

33. Wiederstein M, Sippl MJ. ProSA-web: interactive web service for the recognition of errors in three-dimensional structures of proteins. Nucleic Acids Res. 2007;35:W407-10.

34. Rose PW, Bi C, Bluhm WF, Christie CH, Dimitropoulos D, Dutta S, et al. The RCSB protein data bank: new resources for research and education. Nucleic Acids Res. 2013;41:D475-82

35. Pandurangan AP, Ochoa-Montano B, Ascher DB, Blundell TL. SDM: a server for predicting effects of mutations on protein stability. Nucleic Acids Res. 2017;45:W229-35.

36. Smith RE, Lovell SC, Burke DF, Montalvao RW, Blundell TL. Andante: reducing side-chain rotamer search space during comparative modeling using environment-specific substitution probabilities. Bioinformatics. 2007;23:1099-105.

37. Parthiban V, Gromiha MM, Schomburg D. CUPSAT: prediction of protein stability upon point mutations. Nucleic Acids Res. 2006;34:W239-42.

38. Laimer J, Hiebl-Flach J, Lengauer D, Lackner P. MAESTROweb: a web server for structure-based protein stability prediction. Bioinformatics. 2016:32:1414-6

39. Webb B, Sali A. Comparative protein structure modeling using MODELLER. Curr Protoc Bioinformatics. 2014;47:32.

40. Winter VJ, Cameron A, Tranter R, Sessions RB, Brady RL. Crystal structure of Plasmodium berghei lactate dehydrogenase indicates the unique structural differences of these enzymes are shared across the Plasmodium genus. Mol Biochem Parasitol. 2003;131:1-10.

41. Chaikuad A, Fairweather V, Conners R, Joseph-Horne T, Turgut-Balik D, Brady RL. Structure of lactate dehydrogenase from Plasmodium vivax: complexes with NADH and APADH. Biochemistry. 2005;44:16221-8.

42. Keluskar P, Singh V, Gupta P, Ingle S. Plasmodium falciparum and Plasmodium vivax specific lactate dehydrogenase: genetic polymorphism study from Indian isolates. Infect Genet Evol. 2014;26:313-22.

43. Sundaram B, Varadarajan NM, Subramani PA, Ghosh SK, Nagaraj VA. Purification of a recombinant histidine-tagged lactate dehydrogenase from the malaria parasite, Plasmodium vivax, and characterization of its properties. Biotechnol Lett. 2014:36:2473-80.

44. Hewitt CO, Sessions RB, Dafforn TR, Holbrook JJ. Protein engineering tests of a homology model of Plasmodium falciparum lactate dehydrogenase. Protein Eng. 1997;10:39-44.
45. Talman AM, Duval L, Legrand E, Hubert V, Yen S, Bell D, et al. Evaluation of the intra- and inter-specific genetic variability of Plasmodium lactate dehydrogenase. Malar J. 2007;6:140.

46. Neafsey DE, Galinsky K, Jiang RH, Young L, Sykes SM, Saif S, et al. The malaria parasite Plasmodium vivax exhibits greater genetic diversity than Plasmodium falciparum. Nat Genet. 2012;44:1046-50.

47. Dunn CR, Banfield MJ, Barker JJ, Higham CW, Moreton KM, Turgut-Balik D, et al. The structure of lactate dehydrogenase from Plasmodium falciparum reveals a new target for anti-malarial design. Nat Struct Biol. 1996;3:912-5.

48. Huong NM, Davis TM, Hewitt S, Huong NV, Uyen TT, Nhan DH, et al. Comparison of three antigen detection methods for diagnosis and therapeutic monitoring of malaria: a field study from southern Vietnam. Trop Med Int Health. 2002;7:304-8.

49. Palmer CJ, Lindo JF, Klaskala WI, Quesada JA, Kaminsky R, Baum MK, et al. Evaluation of the OptiMAL test for rapid diagnosis of Plasmodium vivax and Plasmodium falciparum malaria. J Clin Microbiol. 1998;36:203-6.

50. Piper R, Lebras J, Wentworth L, Hunt-Cooke A, Houze S, Chiodini P, et al. Immunocapture diagnostic assays for malaria using Plasmodium lactate dehydrogenase (pLDH). Am J Trop Med Hyg. 1999;60:109-18.

51. Fogg C, Twesigye R, Batwala V, Piola P, Nabasumba C, Kiguli J, et al. Assessment of three new parasite lactate dehydrogenase (pan-pLDH) tests for diagnosis of uncomplicated malaria. Trans R Soc Trop Med Hyg. 2008;102:25-31.

52. Valea I, Tinto H, Nikiema M, Yamuah L, Rouamba N, Drabo M, et al. Performance of OptiMAL-IT compared to microscopy, for malaria detection in Burkina Faso. Trop Med Int Health. 2009;14:338-40.

53. Singh N, Valecha N, Nagpal AC, Mishra SS, Varma HS, Subbarao SK. The hospital- and field-based performances of the OptiMAL test, for malaria diagnosis and treatment monitoring in central India. Ann Trop Med Parasitol. 2003;97:5-13.

54. Golassa L, Enweji N, Erko B, Aseffa A, Swedberg G. Detection of a substantial number of sub-microscopic Plasmodium falciparum infections by polymerase chain reaction: a potential threat to malaria control and diagnosis in Ethiopia. Malar J. 2013;12:352.

55. Daniele S, Giacomelli C, Zappelli E, Granchi C, Trincavelli ML, Minutolo F, et al. Lactate dehydrogenase-A inhibition induces human glioblastoma multiforme stem cell differentiation and death. Sci Rep. 2015;5:15556.

56. Bryant C, Voller A, Smith MJ. The incorporation of radioactivity from (14C) Glucose into the soluble metabolic intermediates of malaria parasites. Am J Trop Med Hyg. 1964;13:515-9.

57. Scheibel LW, Pflaum WK. Carbohydrate metabolism in Plasmodium knowlesi. Comp Biochem Physiol. 1970;37:543-53.

58. Vander Jagt DL, Hunsaker LA, Campos NM, Baack BR. D-Lactate production in erythrocytes infected with Plasmodium falciparum. Mol Biochem Parasitol. 1990;42:277-84.

59. Padmanaban G, Nagaraj VA, Rangarajan PN. An alternative model for heme biosynthesis in the malarial parasite. Trends Biochem Sci. 2007;32:443-9.

60. Jang JW, Cho CH, Han ET, An SS, Lim CS. pLDH level of clinically isolated Plasmodium vivax and detection limit of $\mathrm{pLDH}$ based malaria rapid diagnostic test. Malar J. 2013;12:181.

\section{Publisher's Note}

Springer Nature remains neutral with regard to jurisdictional claims in published maps and institutional affiliations. 